\title{
Wie hoch darf der Blutdruck bei Diabetikern sein?
}

\begin{abstract}
Durch ACCORD und andere Studien ist die Diskussion um den Zielblutdruck bei Hypertoniepatienten mit Diabetes mellitus seit einiger Zeit neu entbrannt. Nun befasste sich eine weitere Studie mit dem Zielblutdruck für dieses spezielle Patientenkollektiv. Die Ergebnisse sprechen wieder für eine strenge Blutdruckeinstellung.
\end{abstract}

_ Die vorliegende Arbeit hat an 35041 Patienten mit Hypertonie und Typ-2Diabetes mellitus unter antihypertensiver Behandlung und 18512 unbehandelten Patienten ohne vorbekannte Herzinsuffizienz die kardiovaskulären Ereignisse innerhalb von sechs Jahren beobachtet. Die Daten wurden dem Nationalen Schwedischen Diabetesregisters entnommen.

Für einen systolischen Blutdruck von $140 \mathrm{mmHg}$ und höher sowie für einen diastolischen Blutdruck von $80 \mathrm{mmHg}$ und höher waren das Risiko für kardiovaskuläre Ereignisse, KHK und Schlaganfall sowie Gesamtmortalität signifikant erhöht. Für den Schlaganfall gab es weder für den systolischen noch für den diastolischen Blutdruck ein J-KurvenPhänomen, $d$. h. bei niedrigeren Blutdruckwerten bis $100 \mathrm{mmHg}$ systolisch oder $60 \mathrm{mmHg}$ diastolisch stieg die Häufigkeit von Schlaganfällen nicht an im Vergleich zu Patienten mit höherem systolischen Blutdruck.

Für die koronare Herzerkrankung und die gesamten kardiovaskulären Ereignisse fand sich im Bereich des systolischen Blutdrucks zwischen 100 und $114 \mathrm{mmHg}$ eine höhere Inzidenz.

\section{Kommentar}

Die Ergebnisse sind im Rahmen der Diskussion um den Zielblutdruck interessant. Diese Befunde weisen darauf hin, bei allen Einschränkungen durch das Studiendesign, dass hypertensive Typ-2-Diabetiker mit Blutdruckwerten deutlich unter $140 \mathrm{mmHg}$ systolisch und unter $80 \mathrm{mmHg}$ diastolisch eine bessere kardiovaskuläre Prognose haben als mit Blutdruckwerten über 140/80 $\mathrm{mmHg}$. Auch niedrigere Blutdruckwerte waren nicht mit einer schlechteren kardiovaskulären Prognose assoziiert. Erst im Blutdruckbereich zwischen 100 und $114 \mathrm{mmHg}$ systolisch war die kardiovaskuläre Prognose schlechter. Wenngleich damit die Diskussion um den optimalen Zielblutdruck bei Hypertoniepatienten mit Diabetes nicht abgeschlossen ist, sprechen auch diese Befunde für eine strenge Blutdruckeinstellung beim hypertensiven Typ-2-Diabetiker.

W. ZIDEK =
- J. Cederholm et al.

Blood pressure and risk of cardiovascular diseases in type 2 diabetes: further findings from the Swedish National Diabetes Register (NDR-BPII). J. Hypertens. 2012, DOI:10.1097/ HJH.0b013e3283577bdf

\section{Tiefe Beinvenenthrombosen als Zeitbombe}

\begin{abstract}
Tiefe Beinvenenthrombosen haben unmittelbar nach dem Ereignis eine hohe Mortalität. Wie sieht aber die Langzeitprognose aus.
\end{abstract}

- In einer Fall-Kontroll-Studie mit 4947 Patienten im Alter zwischen 18 und 70 Jahren, die alle eine erste nicht tödliche venöse Thromboembolie (VTE) durchgemacht hatten, wurde die mittelfristige Mortalität über einen Zeitraum von bis zu acht Jahren untersucht (Holländisches Sterberegister). Die mittlere Beobachtungszeit betrug 5,5 Jahre. Den 4947 Patienten wurde eine Kontrollgruppe mit 6154 Personen ohne stattgehabter Thrombose im Alter zwischen 18 und 70 Jahren gegenübergestellt.

Im Beobachtungszeitraum starben 736 Studienteilnehmer (601 VTE-Patienten und 135 Kontrollpersonen). Die

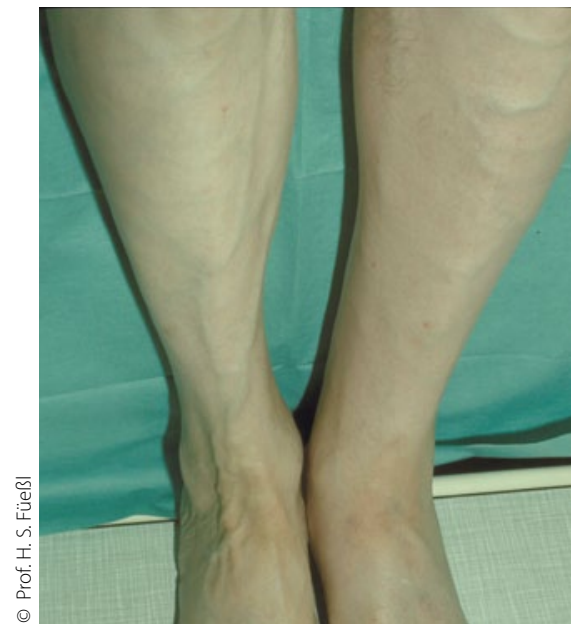

Tiefe Beinvenenthrombose links.

Sterblichkeit war in der VTE-Gruppe mit 22,7 im Vergleich zu 4,7 Todesfällen pro 100 Personenjahre um den Faktor 4 erhöht. Es zeigte sich, dass die Sterblich- keit nach einer tiefen Beinvenenthrombose noch jahrelang deutlich erhöht war.

\section{Kommentar}

Diese Studie zeigt, dass Patienten mit einer tiefen Beinvenenthrombose über viele Jahre hinweg eine deutlich erhöhte Mortalität aufweisen. Im Durchschnitt wurde die Lebenserwartung durch ein thromboembolisches Ereignis ohne zugrundeliegende Krebserkrankung um fünf Jahre gesenkt (von 81 auf 76 Jahre bei Männern und von 84 auf 79 Jahre bei Frauen). Die Studie zeigt, dass man Thrombosepatienten über Jahre hinweg gut im Auge behalten sollte.

C. DIEHM =

\section{- L. E. Flintermann et al.}

Long-Term Survival in a Large Cohort of Patients with Venous Thrombosis: Incidence and Predictors. PLoS Med 2012;9:e1001155. doi:10.1371/journal.pmed.1001155 